\title{
A rank-and-compare algorithm to detect abnormally low bids in procurement auctions
}

\author{
Pier Luigi Conti ${ }^{\mathrm{a}}$, Livia De Giovanni ${ }^{\mathrm{b}}$, Maurizio Naldi ${ }^{\mathrm{c}, *}$ \\ ${ }^{a}$ Università di Roma La Sapienza, Dipartimento di Scienze Statistiche, P.le Aldo Moro 5, 00185 Rome, Italy \\ b Università LUISS “Guido Carli”, Dipartimento di Scienze Politiche, Rome, Italy \\ ' Università di Roma “Tor Vergata", Dipartimento di Informatica, Sistemi e Produzione, Via del Politecnico 1, 00133 Rome, Italy
}

\section{A R T I C L E I N F O}

\section{Article history:}

Received 18 January 2011

Received in revised form 30 December 2011

Accepted 30 December 2011

Available online 11 January 2012

\section{Keywords:}

Auctions

Procurement

Abnormally low bids

Courtesy bids

Bidding behavior

Detection algorithm

Economic theory

\begin{abstract}
A B S T R A C T
Detecting abnormally low bids in procurement auctions is a recognized problem, since their acceptance could result in the winner not being able to provide the service or work awarded by the auction, which is a significant risk for the auctioneer. A rank-and-compare algorithm is considered to detect such anomalous bids and help auctioneers in achieving an effective rejection decision. Analytical expressions and simulation results are provided for the detection probability, as well as for the false alarm probability. The suggested range of application of the detection algorithm leaves out the cases of many tenderers (more than 20) and quite dispersed bids (coefficient of variation larger than 0.15). An increase in the number of tenderers leads to contrasting effects, since both the false alarm probability and the detection probability are reduced. If the bids are spread over a large range, we have instead a double negative effect, with more false alarms and less detections. The presence of multiple anomalous bids worsens the performance of the algorithm as well. On the other hand, the method is quite robust to the presence of courtesy bids.
\end{abstract}

(c) 2012 Elsevier B.V. All rights reserved.

\section{Introduction}

Auctions are a widespread tool for the assignment step in the procurement process (Dimitri et al. 2006, Banker and Mitra 2007, Sardinha et al. 2009). Traditionally, procurement is accomplished primarily through electronic means, and represents now a major example of B2B e-commerce activities (Davila et al. 2003, Subramaniam and Shaw 2002). Electronic procurement auctions can boast advantages that go beyond mere cost savings and include improvements in the cycle time, enhancement of quality, and rationalization of the supply base (Amelinckx et al. 2008), which can be further enhanced by incorporating quality negotiations in the procurement mechanism (Huang et al. 2011). In procurement auctions, a customer (e.g., the government or a company) asks potential suppliers to submit their own bids to provide some specified goods and services. Each bid comes with the price the supplier will charge the customer for the provision of those goods and services. Procurement auctions represent an instance of reverse auctions, since we have a single buyer and multiple sellers. In direct auctions the seller manages the auction and seeks to maximize its profit by choosing the buyer submitting the highest bid; in procurement auctions the buyer manages the auction and seeks

\footnotetext{
* Corresponding author.

E-mail addresses: pierluigi.conti@uniroma1.it (P.L. Conti), ldegiovanni@luiss.it (L. De Giovanni), naldi@disp.uniroma2.it (M. Naldi).
}

to minimize its expense by choosing the seller submitting the lowest bid. It is generally assumed that each prospective seller submits a bid as small as possible, but compatible with its own costs and expected rate of return.

However, bidders may deviate from this rational behavior, and submit bids termed in the literature as anomalous. Namely, they may submit bids that are either too small or too high when compared to the bidders' costs and expected rate of return.

The former case is known as the phenomenon of abnormally low bids; it has been recognized since long and has spurred the European Union to signal it (EUWG 1999). A number of reasons may exist for such anomalous behavior: the bidder may be in desperate need of a contract, though it may turn into a financial loss; it may lack experience in auctions; it may miscalculate its costs and the rate of return needed to repay its funding sources (Gunduz and Karacan 2009). In other cases, it may deliberately submit a low bid to oust a competitor, either to protect its position in the market or to enter a new market (Alexandersson and Hultén 2006, Alexandersson and Hultén 2007). Such a behavior is known as predatory bidding, and represents an instance of the well known phenomenon of predatory pricing (Kobayashi 2008), applied to the context of auctions.

At the same time, bidders could submit bids that are too high with respect to what is expected to win the auction. Such phenomenon has been known for a long time as well (McCaffer 1976, Whittaker 1970). Again, a number of reasons may be considered 
for this behavior: the buyer may have little interest in the contract for sale, or lack the resources and skills to properly submit a suitable bid, or submit just to remain considered for future sales (Skitmore 2002). The resulting bids are often named courtesy bids.

Though both courtesy bids and abnormally low bids represent an anomalous behavior, the assignment and pricing procedures in procurement auctions are actually altered just by the latter. In fact, since the contract is awarded to the lowest bid, and courtesy bids are not expected to be the lowest ones, their presence does not change the final outcome of the auction. On the other hand, abnormally low bids will often result the lowest in the set of submitted bids and declared winners. Since abnormally low bids are associated, either deliberately or not, to slashed rates of return or even to prices lower than costs, the business deriving from winning an auction through submitting an abnormally low bid may not be sustainable, and the awarded contract may not be honored in the end.

Some mechanisms have been proposed in the literature to prevent the manipulation of auctions. For example, in Porter et al. (2008) an assignment mechanism is proposed that offers a contract to the winning tenderer, but the payment is contingent on whether the task is completed, so reducing the risk for the auctioneer. Another mechanism is proposed by Ramchurn et al. (2009) that takes into account the probability that the contractor actually completes the project and its reputation. In Chen et al. (2010), the assignment includes a penalty to be paid by the contractor if the project does not meet the requirements, and that penalty, proposed by each bidder, is used to rank the tenders. On the other hand, the notion that bidders may cheat may alter the strategies followed by other bidders; the resulting equilibrium strategies have been studied by Porter and Shoham (2005).

Here we consider the case where a plain first price (in this case, the lowest price) mechanism is in place. And, rather than through a preventive mechanism, anomalous bids are detected when they take place. Even in the case of assignment mechanisms aiming at avoiding the probability of success of anomalous tenders, a subsequent phase where surviving anomalous tenders are detected may help in further reducing the risk associated to anomalous behavior.

For those reasons, abnormally low bids should be detected, and the reasons behind them investigated to assess whether they derive from a true competitive advantage or just from situations such as those described above. It is commonly accepted that the rejection of bids cannot be performed in an automated way, but an algorithm is needed to detect suspicious bids and submit them to a thorough investigation. The detection algorithm has therefore to act in the framework of a decision support system, an established tool to aid the auctioneer in auction operations such as this (Papazoglou and Tsalgatidou 2000).

In Statistics, observations that stand outside the bulk of data (i.e., either too small or too large) are named outliers. Hence, both abnormally low bids and courtesy bids could be classified as outliers. Many tests have been proposed in the statistical literature to identify and remove outliers. An excellent book on outliers detection is that by Barnett and Lewis (1994), and good surveys are in Beckman and Cook (1983) and Tietjen (1986); prominent techniques are also described in the seminal paper by Grubbs (1950). In addition, some tests have been devised for the specific purpose of detecting bids due to cover pricing (McCaffer and Pettitt 1976, Skitmore 2002). A number of specific schemes, typically different from the ones above mentioned, have been introduced in public documents for the detection of abnormally low bids. Examples are the national regulations in Spain (Ministerio de Hacienda de España 2000, Ministerio de Hacienda de España 2001), Italy (Presidente della Repubblica Italiana 2006), Germany, and Turkey (Zanza 2004). The introduction of such schemes has not been generally accompanied by a proper evaluation of their performances, namely, of their capability to detect anomalous bids without declaring as anomalous otherwise regular bids (which we may name a false alarm). However, a special class of such schemes, adopted in Spain and Italy, and based on the deviation of the abnormally low bid from the average bid, has been evaluated by Conti and Naldi (2008). It has been shown that its performance heavily depends on the number of tenderers and on the dispersion of bid values, therefore calling for caution in the use of that average-bid criterion, and for a careful choice of the parameter setting the detection threshold.

A new class of detection algorithms has been proposed for abnormally low bids, based on a preliminary sorting of the bids and on the comparison of the lowest bid with the second lowest bid. A particular instance of this algorithm has been incorporated in the German law (Zanza 2004). We name the general form of this class the rank-and-compare algorithm; the single instances of the class differ for the value assigned to a single parameter (the Maximum Allowed Deviation), which acts as a scaling factor for the detection threshold. Though applied in a real context, this class of algorithms has not been examined thoroughly, to the best of the authors' knowledge. In Engel (2005), the criterion has been dismissed through the qualitative consideration that bidders anticipating to be excluded would raise their bids to alter the auction's outcome. On the other hand, in Decarolis (2009), an alternative assignment scheme has been proposed to solve the problem of anomalous bids without resorting to statistical detection algorithms. No quantitative evaluation of the rank-and-compare algorithm has been accomplished so far. It can appear strange that a procedure found its way in the national legislation without having been thoroughly examined. However, a detection algorithm based on order statistics, such as the one we examine in this paper, could be expected to be robust with respect to outliers, certainly more than average-based algorithms. That may have been the rationale for its adoption. After its adoption, the German Federal Procurement Agency (BESCHA) has not released data on the effectiveness of that procedure. So, we miss both a theoretical and an empirical evaluation of rank-and-compare algorithms. We aim to fill that gap, by providing a quantitative evaluation of its performance.

In this paper, we examine this new class of rank-and-compare algorithms. The main results we provide are listed below.

(a) Analytical expressions for both the false alarm probability and the detection probability, when a single abnormally low bid is present;

(b) Simulation results for the detection probability under multiple abnormally low bids;

(c) Simulation results for the false alarm and detection probability when courtesy bids are present in addition to abnormally low bids.

On the basis of the above mentioned results, we show that:

(i) the false alarm probability is unaffected by the presence of multiple abnormally low bids;

(ii) the Maximum Allowed Deviation has to be controlled to very low values (e.g., 10\%) to have acceptable values of the detection probability;

(iii) the detection performance degrades gradually as the number of participants grows;

(iv) the algorithm is relatively robust with respect to the presence of courtesy bids.

The paper is organized as follows. In Section 2, we define the probability model for abnormally low bids. The algorithm we are going to examine is described in Section 3. Its evaluation is accomplished in Section 5, after defining both the performance parameters and the evaluation scenarios in Section 4. The evaluation framework follows that already established in Conti and Naldi 
(2008). In Section 6, we provide some hints on the application of the detection criterion in a real world context. The treatment of abnormally low bids after their detection is dealt with in Section 7.

\section{Probability models for bids}

In order to evaluate the rank-and-compare method to detect abnormally low bids, we need specific probability models for the regular bids, the abnormally low bids, and the courtesy bids. We follow here the approach already adopted in Conti and Naldi (2008) for the same purpose. In this section, we briefly review the literature on probability models for bids in auctions, and recall the models we adopt in this paper.

The most widely adopted model for the probability distribution of bids (regular bids in our case) is the Gaussian one, used by McCaffer and Pettitt (1976) in the context of procurement contracts for buildings and roads, by Pin and Scott (1994) for refurbishment works, and by Skitmore (2002) and Conti and Naldi (2008) for the specific purpose of identifying anomalous bids. Less used models are the Log-normal distribution, adopted by Brown (1966) for leasing contract in the oil business, and the Weibull distribution employed by Oren and Rothkopf (1975) for sequential auctions. In Naldi and D'Acquisto (2008), a different approach is taken: rather than adopting one model, a wide range of models is considered, including the uniform, the triangular, the Gaussian, the exponential, and the Pareto distribution.

In this paper, we adopt the widespread Gaussian model, so that any regular bid is considered as a random variable with probability density function

$f_{X}(x)=\frac{1}{\sigma \sqrt{2 \pi}} \exp \left\{-\frac{(x-\mu)^{2}}{2 \sigma^{2}}\right\}$,

where $\mu$ and $\sigma$ are respectively the expected value and the standard deviation of the bids.

For abnormally low bids, we again follow the approach taken in Conti and Naldi (2008), and assume that they have the same distribution as regular bids, although with a rebated mean value to account for a slashed rate of return on the investment. The probability density function for abnormally low bids is therefore

$g_{X}(x)=\frac{1}{\sigma \sqrt{2 \pi}} \exp \left\{-\frac{(x-\beta \mu)^{2}}{2 \sigma^{2}}\right\}$,

where $0<\beta<1$ is the rebating factor. Expected values for the rebating factor can be estimated from an analysis of profits in the industrial sector of interest: the rebating factor could be so low as to zero out the company's expected rate of return on the investment. In a sample case concerning maintenance of public housing, the bids were reported to be $25-33 \%$ higher than the suspected bid, implying a rebating factor in the 0.75-0.8 range (Beresford-Jones 2010).

Finally, for courtesy bids, which are fake bids expected to mimic regular bids (but with an expected value so high not to be competitive), we again follow in the steps of Conti and Naldi (2008) and consider the same distribution as regular bids, although with a (considerably) larger mean value, so that the probability density function is

$h_{X}(x)=\frac{1}{\sigma \sqrt{2 \pi}} \exp \left\{-\frac{(x-\gamma \mu)^{2}}{2 \sigma^{2}}\right\}$,

where $\gamma>1$ is the overbidding factor.

\section{The rank-and-compare criterion}

In this section, we describe the rank-and-compare detection algorithm.
Let $\left\{X_{1}, X_{2}, \ldots, X_{N}\right\}$ be a set of $N$ bids. We can rank them in ascending order, and obtain the ordered set $\left\{X_{(1)}, X_{(2)}, \ldots, X_{(N)}\right\}$, with $X_{(i)} \leqslant X_{(i+1)}$, and $i=1,2, \ldots, N-1$. The lowest bid $X_{(1)}$ in this set is declared to be abnormally low if the inequality

$X_{(1)}<(1-\alpha) X_{(2)}$

holds, where $0<\alpha<1$, and $X_{(2)}$ is the second lowest bid. Since the criterion embodied by Eq. (4) is tantamount to setting a limiting threshold on the maximum deviation from the second lowest bid, we will refer to $\alpha$ as the Maximum Allowed Deviation (MAD). This criterion has been adopted by the Central Purchasing Body of Germany (Zanza 2004), with $\alpha=0.2$.

The rationale behind this criterion is that the lowest bid (which represents the best bid in procurement auctions) is the obvious candidate for investigation of anomalies, and that the degree of anomaly is larger the larger the deviation of the suspected bid from the other bids. With respect to the deviation-from-average criterion investigated by Conti and Naldi (2008), Eq. (4) has the advantage of making the detection threshold insensitive to the abnormally low bid itself: in the deviation-from-average criterion the abnormally low bid influences the average (hence, the detection threshold), by pulling it down.

\section{Performance criteria}

The problem of detecting an abnormally low bid can be expressed as a statistical hypothesis testing problem, where the null hypothesis to be tested is the absence of abnormally low bids. In order to evaluate the detection capability of the rank-and-compare algorithm, we have to introduce a proper set of performance parameters. In this section, we define the performance evaluation problem and the parameters we are going to use, and set the scenarios employed in the evaluation.

\subsection{Performance parameters}

By using the rank-and-compare algorithm, a decision is made whenever a set of bids is submitted to the auction. The outcome of the algorithm is that either the presence of an abnormally low bid is declared or all bids are declared as regular. The nature of the bids can be similarly represented by two alternative situations. Either all bids are regular, or there is at least an abnormally low bid. We can therefore formulate two alternative hypotheses on the nature of bids, denoted by $H_{0}$ (all the bids are actually regular) and $H_{1}$ (there is at least an abnormally low bid). It is to be stressed that hypothesis $H_{1}$ is simply the complement of $H_{0}$. In this sense, and exactly as in outlier detection problems, $H_{1}$ is not crisp, since it contains several possible alternative models for the presence of anomalous bids. At any rate, in evaluating the performance of the detection algorithm considered in the present paper, the model under $H_{1}$ will be precisely specified (Scenarios B-E in Section 4.2).

Accordingly, we can make two different decisions, denoted by $D_{0}$ (all bids are declared regular on the basis of available data) and $D_{1}$ (the presence an abnormally low bid is declared), respectively. The combination of hypotheses and decisions leads to five alternative cases. We are right in two of them:

c1. all bids are regular ( $H_{0}$ true), and no anomalous bid is detected $\left(D_{0}\right.$ correct);

c2. some bids are anomalous $\left(H_{1}\right.$ true) and one of them is correctly identified as anomalous $\left(D_{1}\right.$ correct).

Hence, $D_{1}$ means that the lowest bid $X_{(1)}$ satisfies Eq. (4) and is identified as an abnormally low bid. Instead, the notation $D_{1}$ correct means the bid $X_{(1)}$ satisfying Eq. (4) is actually an abnormally low bid, so that it is correctly identified. In fact, the abnormally low 
bid may not be the minimum in the set of submitted bids, while a regular bid accidentally is the minimum and lies below the threshold, so that the presence of an abnormally low bid is declared (right), but a regular bid is identified as the anomalous one (wrong).

Similarly, we can make wrong decisions in the following three cases:

w1. all bids are regular $\left(H_{0}\right.$ true) and one of them is detected as anomalous ( $D_{1}$ wrong);

w2. some bids are anomalous ( $H_{1}$ true), but all bids are declared regular ( $D_{0}$ wrong);

w3. some bids are anomalous ( $H_{1}$ true) but a regular bid is detected as anomalous ( $D_{1}$ wrong).

In order to evaluate the performance of the rank-and-compare algorithm in this framework, it is enough to use two parameters, related to two of the five situations defined above, namely the detection probability $P_{\operatorname{det}}=\mathbb{P}\left[D_{1} \mid H_{1}\right]$ (whose complement to 1 provides the probability of a wrong decision in the presence of anomalous bids), and the false alarm probability $P_{\mathrm{fa}}=\mathbb{P}\left[D_{1} \mid H_{0}\right]$ (whose complement to 1 provides with the probability of correctly declaring the absence of abnormally low bids).

\subsection{Evaluation scenarios}

In Section 5, we will evaluate the performance parameters in five scenarios, labeled in the following by the capital letters A through $\mathrm{E}$, representing different characteristics of the set of bids.

We consider first Scenario A, where all bids are regular: the $N$ bids follow the same normal distribution, and are mutually independent. In symbols, we have $X_{1}, X_{2}, \ldots, X_{N} \sim \mathbb{N}(\mu, \sigma)$. The choice of the Gaussian distribution to represent the bids is well grounded in the literature; a review of the alternative hypothesis and a justification of the choice is reported in Conti and Naldi (2008). We use Scenario A to evaluate the false alarm probability.

In Scenario B, we assume that all bids but one are regular. The mean value of the single abnormally low bid is lower than that of the regular bids by a rebating factor $\beta$ : the smaller the rebating factor, the more aggressive the rebating behavior of the participant submitting the abnormally low bid. Without loss of generality, we label the anomalous bid as $X_{1}$. We have then the single abnormally low bid $X_{1} \sim \mathbb{N}(\beta \mu, \sigma)$, and $N-1$ regular bids $X_{2}, \ldots, X_{N} \sim \mathbb{N}(\mu, \sigma)$. We employ Scenario $B$ to evaluate the detection probability when a single abnormally low bid is present.

The case of multiple abnormally low bids is considered through Scenario $C$. In this case, there are $M$ abnormally low bids and $N$ $-M$ regular bids, namely, $X_{1}, X_{2}, \ldots, X_{M} \sim \mathbb{N}(\beta \mu, \sigma)$ and $X_{M+1}, X_{M+2}, \ldots, X_{N} \sim \mathbb{N}(\mu, \sigma)$. We have set up a different scenario than that of a single abnormally low bid, since the presence of multiple abnormally low bids changes the nature of the comparison embodied in the detection criterion: the algorithm now compares an abnormally low bid to other abnormally low bids rather than to regular bids. Scenario $C$ is used to evaluate the detection probability under multiple abnormally low bids, while the false alarm probability is still evaluated under Scenario A.

Scenarios D and E are devoted to analyze the case of courtesy bids. Since courtesy bids are submitted not to win, but just to show interest, their value is expected to be larger than the regular ones. As stated in Section 2, we assume here, as in Conti and Naldi (2008), that a courtesy bid follows a Gaussian distribution with the same standard deviation as the regular bids, but with a larger expected value, because of the presence of an overbidding factor $\gamma>1$. In Scenario D, we have $N_{c}$ such courtesy bids, so that the set of bids is subdivided into the set $\left\{X_{1}, \ldots, X_{N-N_{c}}\right\}$ of courtesy bids, where $X_{i} \sim \mathbb{N}(\gamma \mu, \sigma)\left(i=1,2 \ldots, N-N_{c}\right)$, and the set $\left\{X_{N-N_{c}+1}, \ldots, X_{N}\right\}$ of regular bids, where $X_{j} \sim \mathbb{N}(\mu, \sigma)\left(j=N-N_{c}+1, \ldots, N\right)$. Scenario D is used to evaluate the false alarm probability when courtesy bids are present. In order to evaluate the detection probability when courtesy bids are present, we consider instead Scenario E, where both abnormally low and abnormally high bids are present. In such a scenario we have $M$ abnormally low bids, $N_{c}$ courtesy bids, and $N-M-N_{c}$ regular bids; of course, this scenario is set so that the condition $M+N_{c}<N$ holds. We have then $X_{1}, \ldots, X_{M} \sim \mathbb{N}(\beta \mu, \sigma)$, $X_{M+1}, \ldots, X_{N-N_{c}} \sim \mathbb{N}(\mu, \sigma)$, and $X_{N-N_{c}+1}, \ldots, X_{N} \sim \mathbb{N}(\gamma \mu, \sigma)$.

\section{Performance analysis}

\subsection{False alarm probability}

In Section 4, we have defined the false alarm probability as the probability of declaring an abnormally low bid when all the bids are actually regular. In this section, we report the expression for the false alarm probability, and examine its dependence on the auction parameters.

According to Scenario A described in Section 4.2, we assume that there are $N$ regular bids $\left\{X_{1}, X_{2}, \ldots, X_{N}\right\}$, i.i.d. and following a Gaussian distribution with mean $\mu$ and standard deviation $\sigma$. We sort them in ascending order, obtaining an ordered set of variables $\left\{X_{(1)}, X_{(2)}, \ldots, X_{(N)}\right\}$. We obtain the false alarm probability by combining the expressions defined in Section 4.1 and Eq. (4):

$P_{\mathrm{fa}}=\mathbb{P}\left[D_{1} \mid\right.$ Scenario $\left.A\right]=\mathbb{P}\left[X_{(1)}<(1-\alpha) X_{(2)} \mid\right.$ Scenario $\left.A\right]$,

where Scenario A (equivalent to the null hypothesis $H_{0}$ ) is that all bids are regular.

The full derivation of the false alarm probability is reported in Appendix A. We obtain the final expression

$$
\begin{aligned}
P_{\mathrm{fa}} & =N(N-1) \sum_{k=0}^{N-2}\left(\begin{array}{c}
N-2 \\
k
\end{array}\right)(-1)^{N-2-k} \frac{\left[F_{Z}(-\mu / \sigma)\right]^{N-k}}{N-k} \\
& +N(N-1) \int_{F_{Z}(-\mu / \sigma)}^{1}(1-v)^{N-2} F_{Z}\left[(1-\alpha) F_{Z}^{-1}(v)-\alpha \mu / \sigma\right] d v,
\end{aligned}
$$

where the standard Gaussian distribution function $F_{Z}(\cdot)$ has been used.

We can now use the formula for the false alarm probability to examine its dependence on the parameters involved. We first examine its dependence on the relative spread of the bids (embodied in the coefficient of variation of the distribution of bids), and the number $N$ of participants (typical values range from 3 to 12 participants (Albano et al. 2006)). In Fig. 1, where the value $\alpha=0.2$ is used, we see that the maximum tolerable value for the coefficient of variation is approximately $0.1-0.15$; otherwise, the false alarm probability becomes too large and makes the detection criterion unreliable. As expected, the detection criterion is less prone to false alarms as

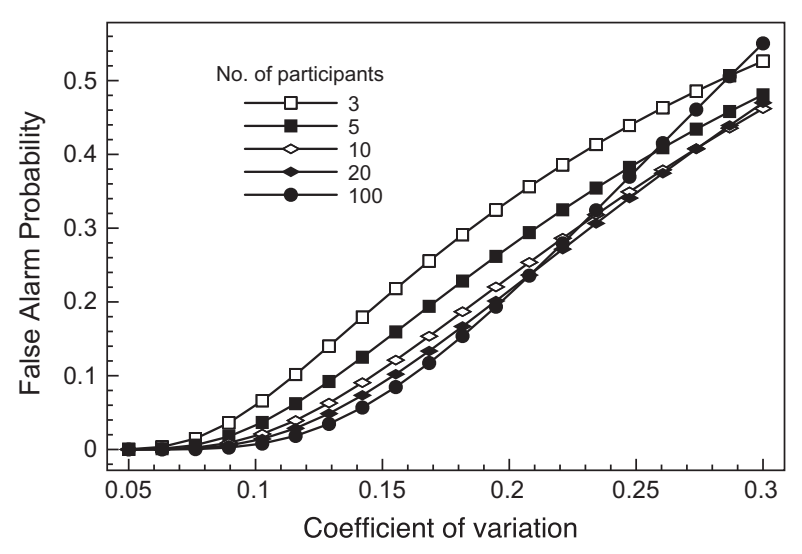

Fig. 1. Impact of the spread of bids on false alarms. 


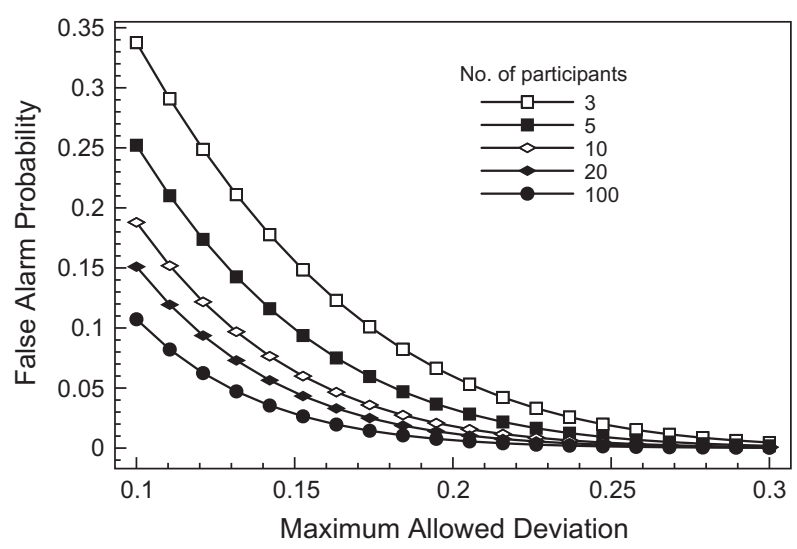

Fig. 2. Impact of the Maximum Allowed Deviation on false alarms.

the number of participants gets larger, as long as the spread of bid values is limited. However, the improvement is practically negligible as $N$ becomes large: there is a very small difference between the curve for $N=20$ participants and that for $N=100$, when the coefficient of variation is smaller than 0.2 . Instead, when both the dispersion of bids gets large and there are a large number of participants (roughly more than 10), the false alarm probability tends to increase with the number of participants.

We can exploit our expression of the false alarm probability to set the correct value for the single parameter involved in the detection criterion, the Maximum Allowed Deviation $\alpha$. In fact, in Fig. 2, plotted for $\sigma / \mu=0.1$, we see how the value of $\alpha$ influences the false alarm probability. As we could expect, the greater the value of $\alpha$ the smaller the false alarm probability, since Eq. (4) is less likely to be satisfied. After setting the desired value for the false alarm probability, we can read the value for the detection threshold on Fig. 2: for any desired value of the false alarm probability, the threshold can be made tighter (allowing just a small deviation from the second lowest bid) when the number of participants gets larger.

\subsection{Detection probability}

After having examined the false alarm probability, we now turn to the capability of the detection algorithm to determine the presence of an anomalous bid. In order to evaluate the detection probability, we consider Scenario B, as set in Section 4.2. We assume that there is a single abnormally low bid. Without loss of generality, we further assume that the abnormally low bid is represented by the random variable $X_{1}$, so that the random variables $X_{i}$ s are mutually independent, with $X_{1} \sim \mathbb{N}(\beta \mu, \sigma)$ and $X_{2}, X_{3}, \ldots, X_{N} \sim \mathbb{N}(\mu, \sigma)$. Our goal is to compute the probability of a $D_{1}$ decision under Scenario $\mathrm{B}$ (detection probability).

The detection probability, denoted by $P_{\text {det }}$, is:

$P_{\text {det }}=\mathbb{P}\left[D_{1}\right.$ correct $\mid$ Scenario $\left.B\right]=\mathbb{P}\left[X_{1}<(1-\alpha) Y\right]$

where $Y=\min \left\{X_{2}, \ldots, X_{N}\right\}$.

The full derivation of the detection probability is provided in Appendix B. We obtain the final expression

$P_{\text {det }}=\int_{0}^{1} F_{Z}\left[(1-\alpha-\beta) \frac{\mu}{\sigma}+(1-\alpha) F_{Z}^{-1}\left(1-(1-v)^{\frac{1}{N-1}}\right)\right] d v$.

Eq. (8) provides that probability that an abnormally low bid is correctly detected and identified. However, as stated in Section 4.1, the straightforward application of the criterion may lead to a regular bid being declared as anomalous in the place of the present truly anomalous bid. In Appendix C, we show that we can be safe with the assumption that the anomalous bid, when present, is

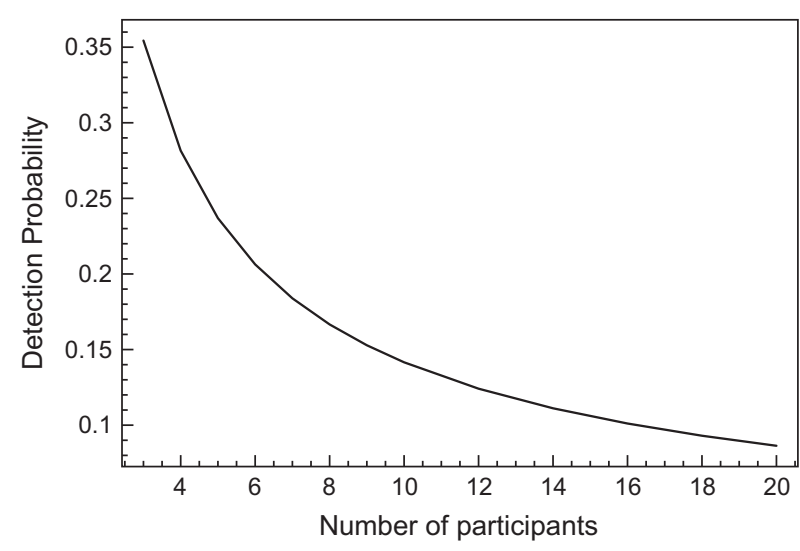

Fig. 3. Impact of the number of participants ( $\alpha=0.2, \beta=0.8$ ).

actually the lowest one $\left(X_{1}<Y\right)$, since the opposite case has a very low probability. Namely, we show that the probability of declaring a regular bid as abnormally low, while the truly anomalous bid is unidentified, is very small.

We can now exploit Eq. (8) to compute the detection probability in a number of cases, examining the influence of the parameters involved: the number of participants, the Maximum Allowed Deviation (determining the detection threshold), the spread of bids, and the rebating factor.

We note first that, when the sum of the Maximum Allowed Deviation and the mean rebating factor is $\alpha+\beta=1$, the spread of bids, represented by the coefficient of variation $\sigma / \mu$, does not affect the detection probability.

We examine then the influence of the number of participants in this special case. The resulting detection probability is plotted in Fig. 3. As expected, the detection probability lowers when the number of participants grows. In fact, in Scenario B we have a single anomalous bid and a growing number of regular bid. When the number of regular bids grows, the minimum among them decreases, so that it becomes closer and closer to the anomalous bid.

For the same reason, the detection probability gets smaller as the standard deviation of the bids increases. This conclusion is evident from Fig. 4, where the detection threshold is set at $20 \%$ less than the second lowest bid, and the rebating factor of the abnormally low bid is $30 \%$. Again, the effect is stronger when the number of participants increases.

The single abnormally low bid is easier to detect if it is much smaller than regular bids. This feature of the abnormally low bid is expressed through its rebating factor $\beta$ : the smaller the rebating factor $\beta$, the smaller the mean value of the abnormally low bid. The

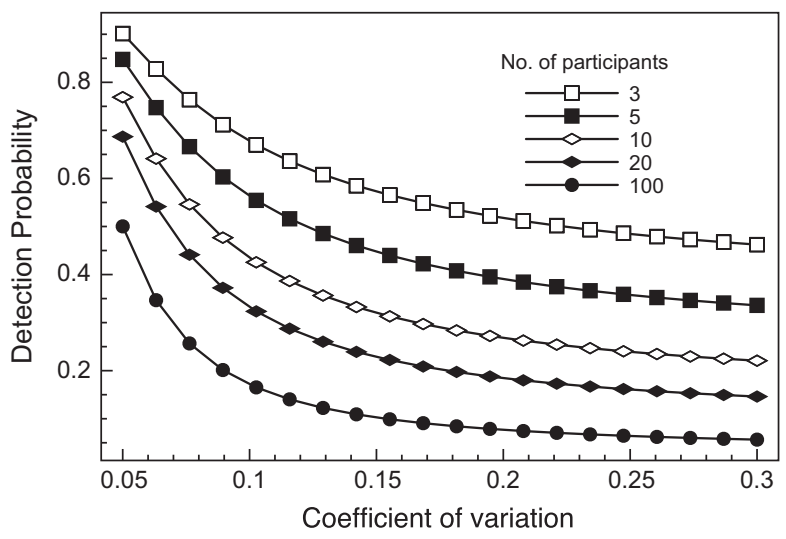

Fig. 4. Impact of the spread of bids on the detection probability $(\alpha=0.2, \beta=0.7$ ). 


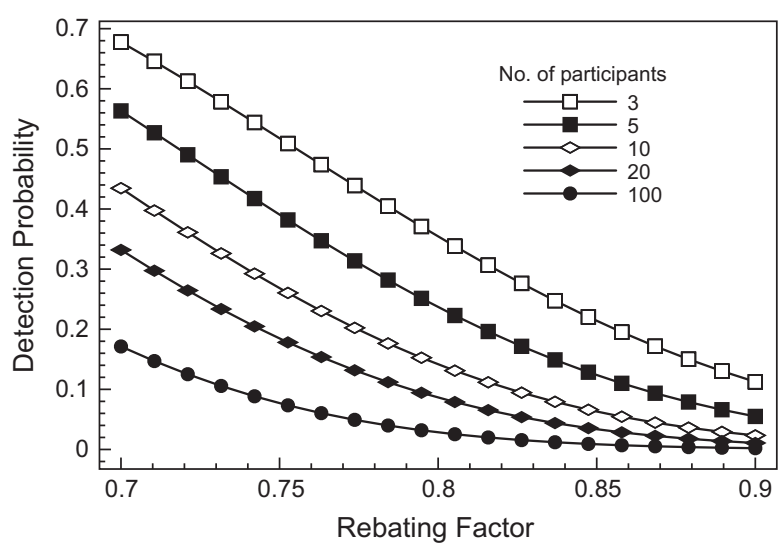

Fig. 5. Impact of the rebating factor on the detection probability $(\alpha=0.2, \sigma / \mu=0.1)$.

behavior of the detection algorithm in this case is described by the curves in Fig. 5. The detection probability decreases as the rebating factor grows. The trend is practically linear when the number of participants is small, but it is still roughly linear for a large range of values of the rebating factor even when there are twenty participants.

Finally, let us consider the effect of the only design parameter, the Maximum Allowed Deviation, driving the detection threshold. Here we set $\sigma / \mu=0.1$ and consider two different values for the rebating factor in Figs. 6 and 7. Being more tolerant, by setting a smaller detection threshold through a larger $\alpha$, has the obvious

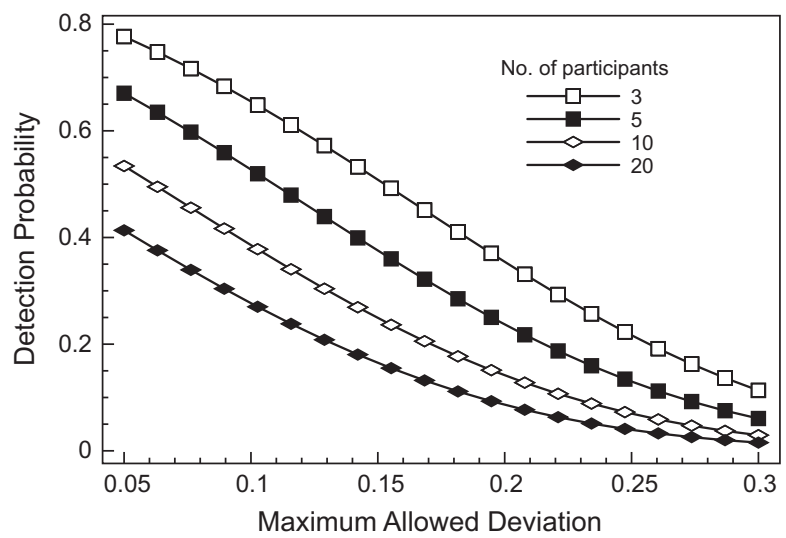

Fig. 6. Impact of the detection threshold $(\sigma / \mu=0.1, \beta=0.8)$.

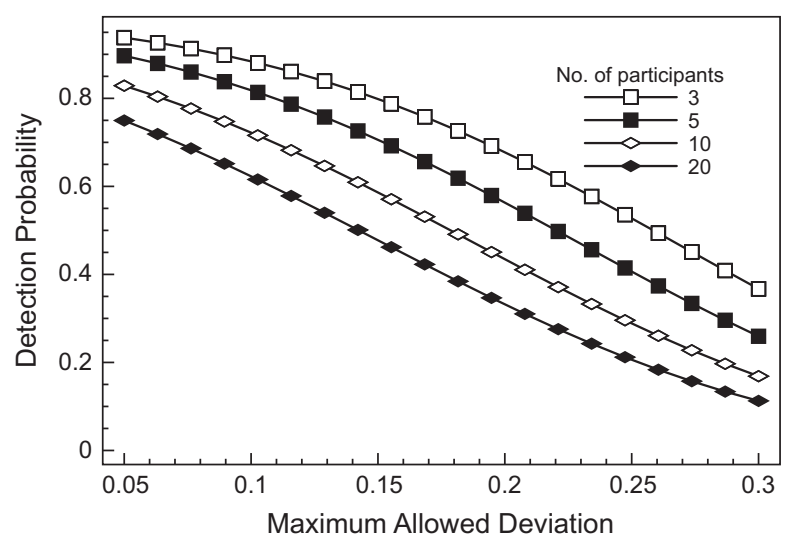

Fig. 7. Impact of the detection threshold $(\sigma / \mu=0.1, \beta=0.7)$. effect of lowering the detection probability. However, the shape of the curves is quite different from the other detection curves seen so far, since the trend is roughly linear in most cases.

\subsection{Detection of multiple abnormally low bids}

We have considered so far the case of a single abnormally low bid. However, it may happen that multiple abnormally low bids are submitted. In this subsection we analyze how the rank-andcompare algorithm performs in those situations, by evaluating both the false alarm probability and the detection probability.

As far as the false alarm probability is concerned, nothing changes with respect to the single abnormally low bid case, since the false alarm probability is evaluated under Scenario A, where all bids are regular.

On the other hand, the detection probability is expected to change, since multiple abnormally low bids may contribute to move the detection threshold. We consider now Scenario $C$, where we have $M$ abnormally low bids and $N-M$ regular bids, with $2 \leqslant M<N$. Because of its very definition, the rank-and-compare algorithm can detect at most a single abnormally low bid. In fact, in the rank-and-compare algorithm, the lowest bid is compared, through Eq. (4), to the second lowest bid, which is then excluded from the number of possible abnormally low bids, though it would be the second best candidate after the lowest bid.

We confine ourselves to the case of (correctly) detecting one abnormally low bid among the existing $M$, namely the minimum among them. If we indicate such minimum by $W=\min \left\{X_{1}\right.$, $\left.X_{2}, \ldots, X_{M}\right\}$, the probability of detecting an abnormally low bid is then

$P_{\mathrm{det}}=\mathbb{P}\left[D_{1}\right.$ correct $\mid$ Scenario $\left.C\right]=\mathbb{P}\left[W<(1-\alpha) X_{(2)}\right]$,

where the order statistics $X_{(2)}$ is drawn among all the $N$ bids (including both the regular ones and the abnormally low ones), and could be either an abnormally low bid, or a regular one. We do not assume here the condition $\max \left(X_{1}, X_{2}, \ldots, X_{M}\right)<\min \left(X_{M+1}, \ldots, X_{N}\right)$ that all the abnormally low bids are lower than the regular bids, although that would simplify the analysis. Here, the detection probability could in principle be computed by an approach similar to that of Section 5.2, but we prefer to resort to Monte Carlo simulation. A sample result is shown in Fig. 8, where the curve obtained for a single abnormally low bid is also reported. The detection probability decreases as the number of abnormally low bids increases, but just when the rebating factor is sufficiently small. When the abnormally low bids are not considerably lower than the regular ones (roughly, when the rebating factor is larger than 0.8 ), the presence of multiple abnormally low bids slightly increases the probability of detecting

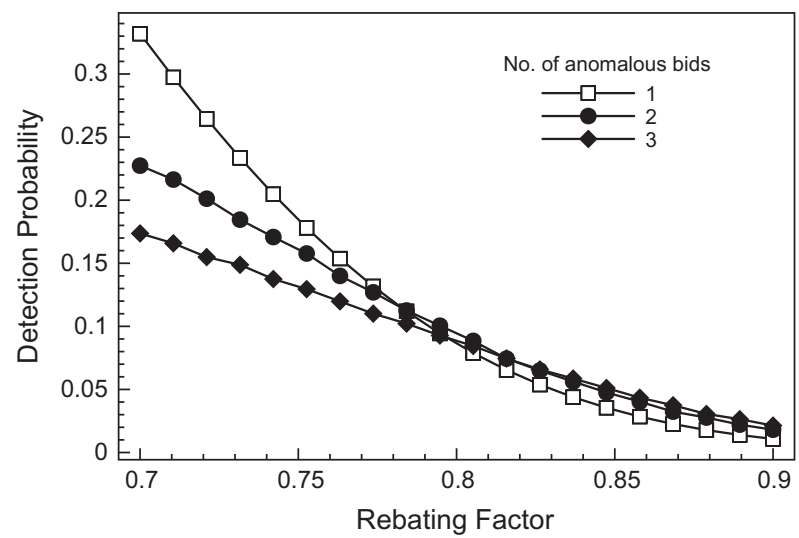

Fig. 8. Detection of multiple abnormally low bids $(\sigma / \mu=0.1, \alpha=0.2, N=20)$. 


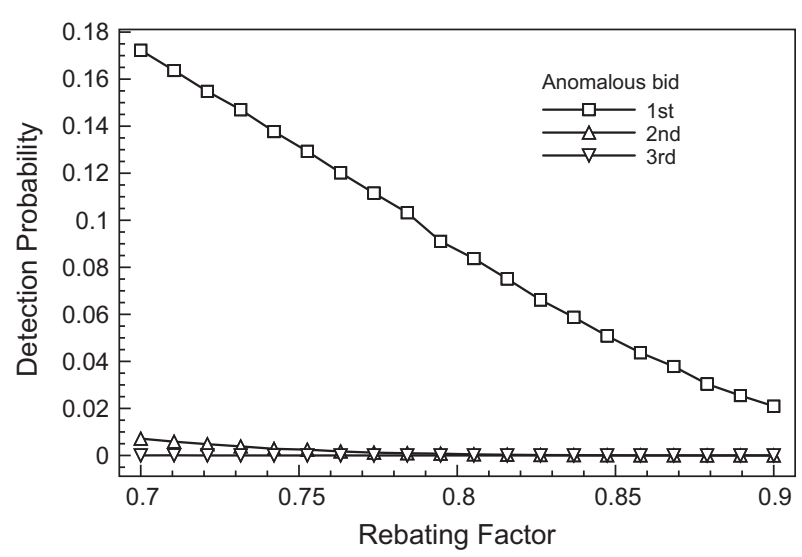

Fig. 9. Detection of three abnormally low bids $(\sigma / \mu=0.1, \alpha=0.2, N=20)$.

one of them. Since $10^{5}$ simulation runs have been performed for each point of the curve, the relative standard error is $1.38 \%$ when the true detection probability is 0.05 and $0.63 \%$ when the true detection probability is 0.2 , which can be considered as virtually negligible over the whole range of cases examined.

A possible way to overcome this problem consists in using the rank-and-compare algorithm iteratively, by comparing the lowest bid with the second lowest bid to detect the first abnormally low bid, and then, if that first detection takes place, comparing the second lowest bid to the third lowest bid to detect a possible second abnormally low bid, and so on, till the first negative comparison. We have evaluated the performance of this iterative approach, again by simulation. We report in Figs. 9 and 10 the results obtained, when there are respectively three and two anomalous bids, under the same conditions adopted for Fig. 8. We see that the capability to detect anomalous bids is quite negligible beyond the first one.

\subsection{Impact of courtesy bids}

As recalled in the Introduction, bids can be anomalous because they are either too high or too small. Bids that are too high are often called courtesy bids. Their presence alters somewhat the correct behavior of auctions. In this section, we evaluate their impact on the false alarm and detection probability.

We consider first the false alarm probability. Courtesy bids may act on the false alarm probability by modifying the detection threshold. In the deviation-from-average criterion studied by Conti and Naldi (2008), courtesy bids directly contribute to the average of the bids submitted (and therefore to the detection threshold).

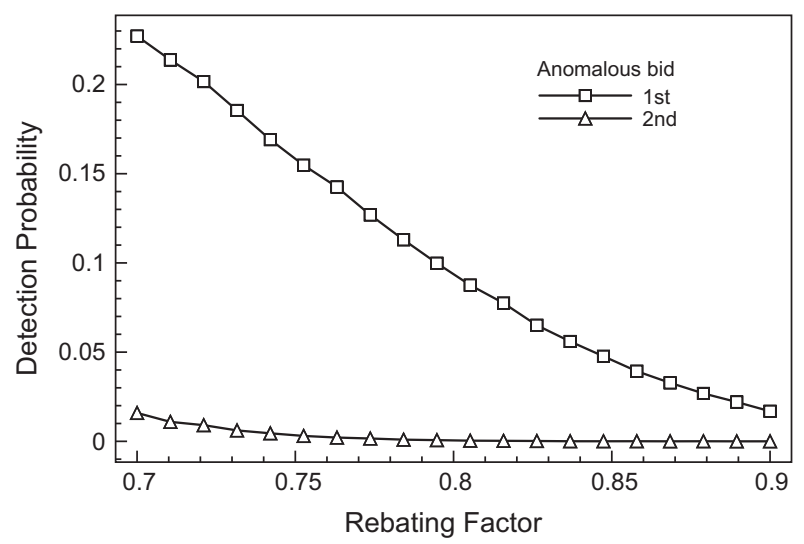

Fig. 10. Detection of two abnormally low bids $(\sigma / \mu=0.1, \alpha=0.2, N=20)$.
Instead, in the rank-and-compare algorithm, a courtesy bid may directly alter the false alarm probability only if it is either the lowest bid or the second lowest one, which are both events with a negligible probability: courtesy bids are unlikely to directly influence the false alarm probability. However, since the presence of courtesy bids in the set of $N$ submitted bids reduces the number of regular bids, the range covered by the sample of regular bids is smaller, and the detection threshold may result to be a bit higher. We examine this effect separately for the cases of a single courtesy bid and multiple courtesy bids.

We adopt Scenario D, as defined in Section 4. When there is a single courtesy bid $\left(N_{c}=1\right)$, and its mean value is significantly larger than that of regular bids $(\gamma \gg 1)$, we can assume that the courtesy bid is anyway larger than the regular bids. If we label the single courtesy bid by $X_{N}$, we have $\max \left\{X_{1}, \ldots, X_{N-1}\right\}<X_{N}$. The detection threshold is then actually set through the second lowest value in a set of $N-1$ regular bids, and we can exploit the expression derived in Section 5.1 for the false alarm probability in the absence of courtesy bids, namely, Eq. (6), by using $N-1$ instead of $N$. The resulting false alarm probability $P_{\mathrm{fac}}$ is

$$
\begin{aligned}
& P_{\mathrm{fac}}=(N-1)(N-2) \sum_{k=0}^{N-3}\left(\begin{array}{c}
N-3 \\
k
\end{array}\right)(-1)^{N-3-k} \frac{\left[F_{Z}(-\mu / \sigma)\right]^{N-k-1}}{N-k-1} \\
& +(N-1)(N-2) \int_{F_{Z}(-\mu / \sigma)}^{1}(1-v)^{N-3} F_{Z}\left[(1-\alpha) F_{Z}^{-1}(v)-\alpha \mu / \sigma\right] d v .
\end{aligned}
$$

In order to evaluate the impact of courtesy bids, the ratio $P_{\mathrm{fac}} / P_{\mathrm{fa}}$ (ratio of the false alarm probability in the presence of a single courtesy bid to the false alarm probability when all the bids are regular) is plotted in Fig. 11. The false alarm probability is expected to increase, because of the presence of the courtesy bid, which raises the detection threshold; the impact is larger for auctions with a small number of participants. As it appears from Fig. 11, when there are just five participants (and one of them submits a courtesy bid) the false alarm probability grows by more than $25 \%$. However, when there are several courtesy bids, or the expected value of the single courtesy bid is not significantly larger than that of the regular bids, we cannot use Eq. (9). The latter case can be dealt with by considering that Eq. (9) represents an upper bound for the false alarm probability when there is a single courtesy bid: when the overbidding factor approaches 1 , we fall back to the case of all regular bids.

In order to analyze the case of multiple courtesy bids, we resort to Monte Carlo simulation. In Fig. 12, the false alarm probability is shown, when there are 20 participants, of which up to five submit courtesy bids. Even in this case (the number of courtesy bids is very large, making up $1 / 4$ of the overall number of participants), the false alarm probability increases by no more than roughly $20 \%$ with respect to the case of all regular bids.

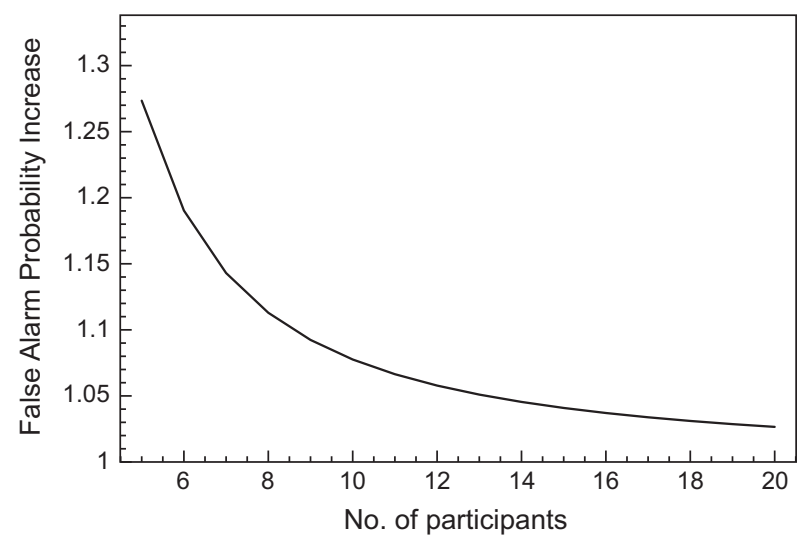

Fig. 11. Increase of false alarms due to a single courtesy bid $(\sigma / \mu=0.1, \alpha=0.2)$. 


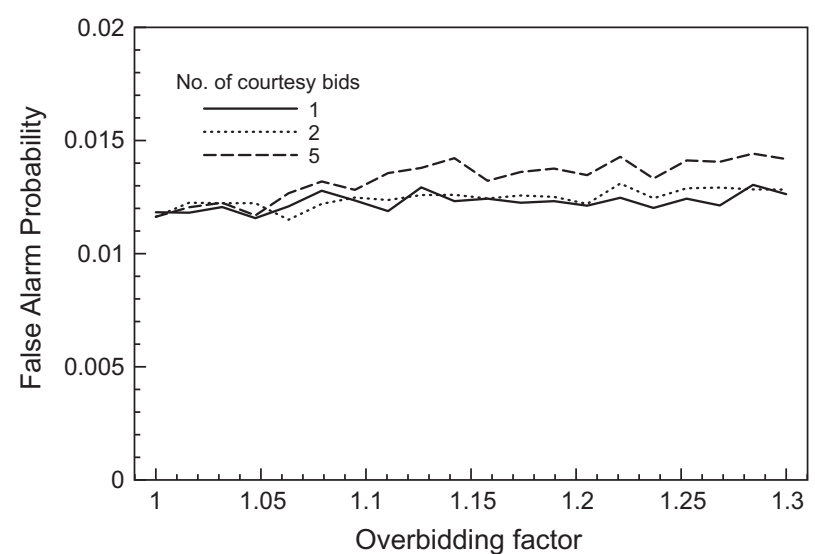

Fig. 12. False alarm probability under multiple courtesy bids $(\sigma / \mu=0.1, \alpha=0.2$, $N=20)$.

We can therefore conclude that the influence of courtesy bids on the false alarm probability is quite limited, and that the rankand-compare algorithm, since it is based on order statistics, provides a built-in robustness against such anomalous behavior.

We now turn to the detection probability, for which we employ Scenario E, as defined in Section 4.2. As in the case of the false alarm probability, the presence of courtesy bids could increase the detection probability. In order to evaluate the extent of this effect, we have resorted again to Monte Carlo simulation, with a sample of $10^{5}$ elements. Four sample curves are shown in Figs. 13 and 14. In those cases, we have set a large number of participants $(N=20)$, a single abnormally low bid, a typical value for the Maximum Allowed Deviation $\alpha=0.2$, and have observed how the detection probability behaves when the overbidding factor grows (the overbidding factor $\gamma=1$ corresponds to the case of no courtesy bids). We expect the detection probability to grow with the overbidding factor, since a larger overbidding factor pulls the detection threshold up. When the rebating factor is quite small ( $\beta=0.7$ ), as in Fig. 13, the effect is quite limited: over the range considered for the overbidding factor $(\gamma \in[1,1.3])$ the detection probability grows by $12.4 \%$ with 5 courtesy bids, but just by $2.6 \%$ when there is a single courtesy bid (the standard error due to simulation is anyway lower than $0.5 \%$, and that the range examined for the overbidding factor amounts to an increase of $30 \%$, from $\gamma=1$ to $\gamma=1.3$ ). In addition, the increase in the detection probability is reached quite early; for large overbidding factors (say, larger than 1.15), the detection probability is largely independent of the exact

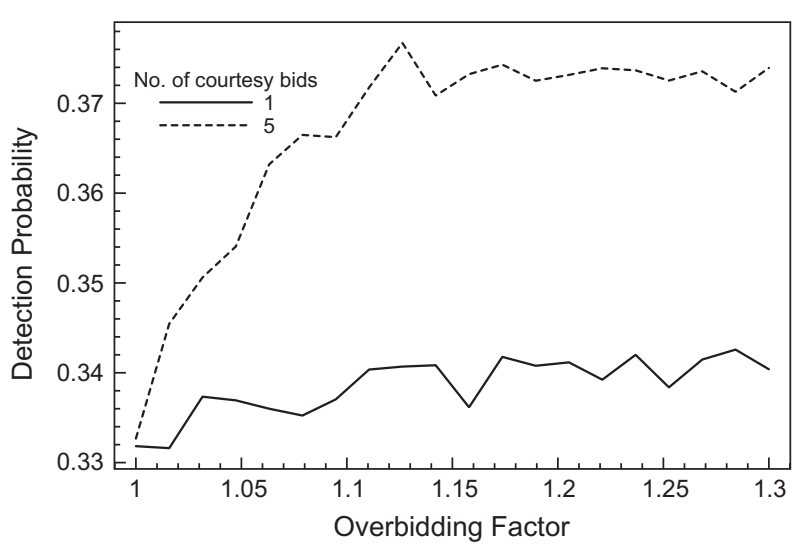

Fig. 13. Detection probability under courtesy bids $(\sigma / \mu=0.1, \alpha=0.2, \beta=0.7$, $N=20)$.

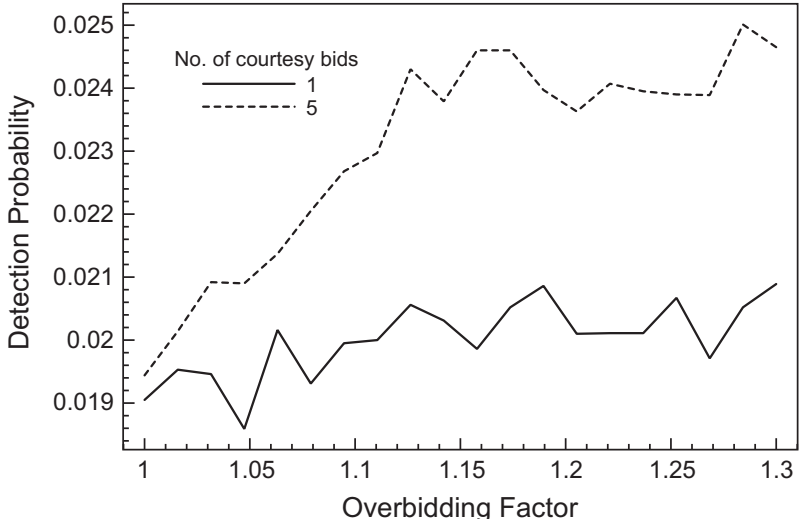

Fig. 14. Detection probability under courtesy bids $(\sigma / \mu=0.1, \alpha=0.2, \beta=0.9$, $N=20$ ).

value of the overbidding factor. This effect becomes more evident as the rebating factor grows. When the abnormally low bids are close to the regular ones (e.g., when $\beta=0.9$ as in Fig. 14), the detection probability increases by $9.7 \%$ even when there is a single courtesy bid, but by a significant $26.8 \%$ when there are 5 courtesy bids (here the increase is roughly equal to that imposed on the overbidding factor). In those cases, the standard error due to simulation is around $2 \%$. We note that the case represented in Fig. 14 is an extreme case, since the rebating factor is quite close to 1 , and the detection probability would anyway be very low, even in the absence of courtesy bids.

We can then conclude that the detection algorithm is relatively robust to courtesy bids, since the impact on the detection probability is significant just when there are at the same time abnormally low bids very close to the regular ones (the rebating factor $\beta$ is close to 1 ), multiple courtesy bids, and large overbidding factors (roughly $\gamma>1.15$ ).

\section{Practical application of the criterion}

In Section 5, we have evaluated the performance of the rankand-compare criterion. The performance analysis tells what happens when the statistical characteristics of the set of bids are known and the detection threshold is set through the Maximum Allowed Deviation. For the practical application of the criterion, we must however set the value of $\alpha$ (the only parameter that needs to be set in this criterion). In this section, we provide some rules of thumb on the basis of the results of the performance analysis.

We have seen that the performance of the algorithm depends strongly on the coefficient of variation of the bids. As the dispersion of the bids gets larger, it becomes progressively difficult to distinguish the abnormally low bids from the regular ones, since a regular bid may exhibit a very small random value. In Section 5.1 , we have seen that the false alarm probability becomes so large to make the algorithm unusable, when the coefficient of variation is larger than 0.15 . That value can therefore be set as a threshold to determine whether to apply this detection criterion at all. However, we do not know in advance the coefficient of variation of the bids. We may estimate its typical values by examining past auctions, or what may be reported in the literature, but those typical values may be different from the case at hand. A first rule of thumb is therefore to preliminarily estimate the coefficient of variation, by computing the sample mean and standard deviation of the bids submitted to the auction of interest. If the estimated coefficient of variation lies above 0.15 , the rank-and-compare criterion should not be applied.

For a similar reason, we should not apply this detection criterion, when the number of participants is too large. Figs. 6 and 7 tell 
us that the detection probability gets progressively lower as the number of participants increases. We should aim at a detection probability not lower than $50 \%$, so that it may not be worthwhile to apply the criterion if there are more than 10-20 participants.

After this preliminary decision, the auctioneer has to set the detection threshold through the Maximum Allowed Deviation $\alpha$. Now, we can set the desired values for false alarm probability and the detection probability, and obtain a suitable range of values for $\alpha$ by plotting curves similar to those shown in Sections 5.1 and 5.2 , but for the values of $\sigma / \mu$ and $N$ at hand. From the results shown so far, it appears that the Maximum Allowed Deviation should be confined to values smaller than 0.15 .

\section{Treatment of abnormally low bids}

So far, we have considered how abnormally low bids can be detected through a statistical test. However, as stressed in the Introduction, the detection task has to be followed by the decision on the fate of the bid suspected to be abnormally low. In this section, we review the possible strategies, their pros and cons, and the direction of the legislation.

After detecting an abnormally low bid, whatever the detection method, the auctioneer may take one of two decisions:

\section{Rejection.}

\section{Quarantine.}

Before examining the two options in greater detail, we note that a private auctioneer is completely free to adopt either strategy 1 or strategy 2. On the other hand, a public auctioneer may be constrained by the laws of its country on public procurement.

In case of rejection, the auctioneer excludes the anomalous bid from the set of candidates that can win the contract, just on the basis of the results of the automatic detection mechanism. This is the usual choice in a number of public procurement contexts, as in Italy, China, Florida (see sub-article $3-2.1$ of the rules set by the Florida Department of Transportation (1997)), and some local governments in Japan (Akai et al. 2009) (a more complete list is contained in Decarolis (2009)). Automatic rejection is a fast decision (and contributes to keep procurement procedures short), and can be the only choice when the auctioning institution has not enough staff to investigate the tenders more thoroughly. Automatic rejection is also considered as a viable choice, even overriding the legislation, when there is a large number of tenders and their detailed examination would be a burden on the contracting authority, as established by the European Court of Justice Cases (2008).

Instead of automatically rejecting the anomalous bid, the auctioneer may decide to examine the tender more closely. In fact, as shown in this paper, the automatic detection mechanism does not completely guarantee that the bid declared as abnormally low is actually anomalous. During the scrutiny, the suspected tender is put into quarantine, and the contract assignment procedure is suspended. For instance, this is the procedure adopted in the European Union (2004), though it may be overridden under special conditions, as stated in the Court of Justice statement recalled above. Several elements of the tender may be considered to justify the low bid, for example:

- the economics of the construction method, the manufacturing process or the services provided;

- the technical solutions chosen and/or any exceptionally favorable conditions available to the tenderer for the execution of the work and for the supply of the goods or services;
- the originality of the work, supplies or services proposed by the tenderer;

- compliance with the provisions relating to employment protection and working conditions in force at the place where the work, service or supply is to be performed;

- the possibility of the tenderer obtaining State aid.

If the tender is abnormally low because the tenderer has obtained State aid, the Directive 2004/18/EC set by the European Parliament Directive (2004) states that the tender can be rejected on that ground alone only after consultation with the tenderer, where the latter is unable to prove, within a time period fixed by the contracting authority, that the aid was legally granted.

A clear advantage of the quarantine solution is that, on the average, it leads to fairer and cheaper procurement, because regular tenders are not unduly rejected, and the resulting prices are lower than those obtained after rejecting the lowest bid. However, it should also be considered that the examination phase may be costly and lengthens the procurement procedure.

\section{Conclusions}

A rank-and-compare method has been evaluated to detect abnormally low bids in procurement auctions. Two performance indices have been selected for its evaluation: the detection probability and the false alarm probability. Their dependence on the number of tenderers, the dispersion of bids, and the rebating factor of the anomalous bid has been investigated.

A large number of tenderers does decrease the detection probability, with a negative effect on the performance of the method. A larger spread of bids as well contributes to reduce the detection probability. This effect is more evident when the number of tenderers is large. The effect of the rebating factor is similar: the higher the rebating factor (which means that the anomalous bid is closer to the regular ones), the smaller the detection probability. Some guidelines for the practical applications of the detection algorithm have been provided, which suggest to perform a preliminary estimation of the dispersion of bids and restrict the application range of the algorithm to the cases where the coefficient of variation of bids is smaller than 0.15 and the number of tenderers is not larger than 20.

We notice that the rank-and-compare method has been designed to detect a single abnormally low bid, but we have also evaluated its performance when used iteratively to detect multiple anomalous bids. Though its performance is not encouraging, its employment in a multiple detection scheme deserves to be further explored in the future, since the detection of bidders bidding consistently low across different auction would represent a hint of colluding behavior.

The impact of courtesy bids (either a single courtesy bid or multiple ones) has also been investigated. When a single courtesy bid is present, both the false alarm probability and the detection probability increase, though the effect is quite moderate. The same effect essentially holds when multiple courtesy bids are present.

\section{Appendix A. Derivation of the false alarm probability}

In this appendix, we derive the expression of the false alarm probability.

We first recall the probability integral transformation (see Th. 2.1.4 in Casella and Berger (1990)), which is used in the following. If $F_{X}(x)$ is the (continuous) cumulative distribution function (c.d.f.) of the random variables (r.v.s) $X_{i}$ s, and if the set of ordered r.v.s $\left\{X_{(1)}, X_{(2)}, \ldots, X_{(N)}\right\}$ is transformed according to $F_{X}(\cdot)$, then an ordered 
sample $\left\{U_{(1)}, U_{(2)}, \ldots, U_{(N)}\right\}$ from a uniform distribution in obtained.

We can now start the computation of the false alarm probability. Using the probability integral transformation and exploiting its monotonicity, which preserves ranking, we get

$$
\begin{aligned}
P_{\mathrm{fa}} & =\mathbb{P}\left[X_{(1)}<(1-\alpha) X_{(2)} \mid \text { Scenario } A\right] \\
& =\mathbb{P}\left[F_{X}^{-1}\left(U_{(1)}\right)<(1-\alpha) F_{X}^{-1}\left(U_{(2)}\right)\right] \\
& =\mathbb{P}\left[F_{X} F_{X}^{-1}\left(U_{(1)}\right)<F_{X}\left((1-\alpha) F_{X}^{-1}\left(U_{(2)}\right)\right)\right] \\
& =\mathbb{P}\left[U_{(1)}<F_{X}\left((1-\alpha) F_{X}^{-1}\left(U_{(2)}\right)\right)\right] .
\end{aligned}
$$

We consider next the joint probability density $f_{U_{(1)} U_{(2)}}(u, v)$ of the two ordered variables $U_{(1)}$ and $U_{(2)}$. Let $\left\{Y_{(1)}, Y_{(2)}, \ldots, Y_{(N)}\right\}$ denote the order statistics of a random sample $\left\{Y_{1}, Y_{2}, \ldots, Y_{N}\right\}$ from a continuous r.v. $Y$, with c.d.f. $F_{Y}(y)$ and density function $f_{Y}(y)$. The joint density function of $Y_{(i)}, Y_{(j)}, 1 \leqslant i<j \leqslant N$ is (cfr. Casella and Berger (1990)).

$$
\begin{aligned}
f_{Y_{(i)} Y_{(i)}}(u, v)= & \frac{N !}{(i-1) !(j-i-1) !(N-j) !} f_{Y}(u) f_{Y}(v) \\
& \times F_{Y}(u)^{i-1}\left[F_{Y}(v)-F_{Y}(u)\right]^{j-i-1}\left[1-F_{Y}(v)\right]^{N-j} .
\end{aligned}
$$

Since the sample is drawn from a uniform distribution in $(0,1)$, $Y_{i}=U_{i}, i=1, \ldots, N$. Taking further into account that $i=1, j=2$, from Eq. (A.2) we obtain

$f_{U_{(1)} U_{(2)}}(u, v)=N(N-1)(1-v)^{N-2}$

From Eq. (A.3), and taking into account that the constraint $U_{(1)}<U_{(2)}$ has to be satisfied, Eq. (A.1) becomes

$$
\begin{aligned}
P_{\mathrm{fa}} & =\int_{0}^{1} \int_{0}^{\min \left[F_{X}\left((1-\alpha) F_{X}^{-1}(v)\right), v\right]} f_{U_{(1)} U_{(2)}}(u, v) d u d v \\
& =\int_{0}^{1} \int_{0}^{\min \left[F_{X}\left((1-\alpha) F_{X}^{-1}(v)\right), v\right]} N(N-1)(1-v)^{N-2} d u d v \\
& =N(N-1) \int_{0}^{1}(1-v)^{N-2} \int_{0}^{\min \left[F_{X}\left((1-\alpha) F_{X}^{-1}(v)\right), v\right]} d u d v . \\
& =N(N-1) \int_{0}^{1}(1-v)^{N-2} \min \left[F_{X}\left((1-\alpha) F_{X}^{-1}(v)\right), v\right] d v .
\end{aligned}
$$

At this point, Eq. (A.4) can be numerically evaluated to get the false alarm probability. However, we can further simplify that expression to get a clearer picture of the relationship between the false alarm probability and the scenario parameters.

In order to find the minimum involved in (A.4), we have to solve the following inequality

$F_{X}\left((1-\alpha) F_{X}^{-1}(v)\right)<v$

By introducing the standard Gaussian cumulative distribution function $F_{Z}(z)$, using its monotonicity, and defining the quantity $z_{v}=F_{Z}^{-1}(v)$ we have first

$$
\begin{aligned}
F_{X}\left((1-\alpha) F_{X}^{-1}(v)\right) & =F_{X}(1-\alpha)\left(\mu+\sigma z_{v}\right) \\
& =F_{Z}\left(\frac{(1-\alpha)\left(\mu+\sigma z_{v}\right)-\mu}{\sigma}\right)
\end{aligned}
$$

frow which the chain of inequalities

$F_{X}\left((1-\alpha) F_{X}^{-1}(v)\right)<v \Longleftrightarrow F_{Z}\left(\frac{(1-\alpha)\left(\mu+\sigma z_{v}\right)-\mu}{\sigma}\right)<F_{Z}\left(z_{v}\right)$

follows, provided that $\mu+\sigma z_{v}>0$, which is of course equivalent to $v>F_{Z}(-\mu / \sigma)$.

The integral in Eq. (A.4) can be then split into two parts, one of which can be easily solved via the binomial theorem, while the other can be computed numerically. In symbols, we have

$$
\begin{aligned}
P_{\mathrm{fa}}= & N(N-1) \int_{0}^{1}(1-v)^{N-2} \min \left[F_{X}\left((1-\alpha) F_{X}^{-1}(v)\right), v\right] d v \\
= & N(N-1) \int_{0}^{1}(1-v)^{N-2} \min \left[F_{Z}\left((1-\alpha) z_{v}-\alpha \mu / \sigma\right), v\right] d v \\
= & N(N-1) \int_{0}^{F_{Z}(-\mu / \sigma)} v(1-v)^{N-2} d v \\
& +N(N-1) \int_{F_{Z}(-\mu / \sigma)}^{1}(1-v)^{N-2} F_{Z}\left[(1-\alpha) z_{v}-\alpha \mu / \sigma\right] d v \\
= & N(N-1) \sum_{k=0}^{N-2}\left(\begin{array}{c}
N-2 \\
k
\end{array}\right)(-1)^{N-2-k} \frac{\left[F_{Z}(-\mu / \sigma)\right]^{N-k}}{N-k} \\
& +N(N-1) \int_{F_{Z}(-\mu / \sigma)}^{1}(1-v)^{N-2} F_{Z}\left[(1-\alpha) F_{Z}^{-1}(v)-\alpha \mu / \sigma\right] d v .
\end{aligned}
$$

\section{Appendix B. Derivation of the detection probability}

In this appendix, we derive the expression of the detection probability.

In order to find out a closed form expression for Eq. (7), let us introduce two auxiliary variables: $U_{1}=F_{X_{1}}\left(X_{1}\right)$, and $U_{Y}=F_{Y}(Y)$. By the probability integral transformation recalled in Appendix $A$, both $U_{1}$ and $U_{Y}$ do have uniform distribution on $[0,1]$. Their use allows us to express the abnormally low bid and the minimum among the regular bids as $X_{1}=F_{X_{1}}^{-1}\left(U_{1}\right)$ and $Y=F_{Y}^{-1}\left(U_{Y}\right)$, respectively. In the latter expression, we need the inverse distribution of the minimum among the $N-1$ regular bids. Since $F_{Y}(y)=1-\left[1-F_{X}(y)\right]^{N-1}\left(F_{X}(\cdot)\right.$ being the common probability distribution function of the regular bids), the inverse expression we need is $Y=F_{X}^{-1}\left[1-\left(1-U_{Y}\right)^{\frac{1}{N-1}}\right]$.

We can now go back to Eq. (7) and obtain

$$
\begin{aligned}
P_{\mathrm{det}} & =\mathbb{P}\left[X_{1}<(1-\alpha) Y\right] \\
& =\mathbb{P}\left[F_{X_{1}}^{-1}\left(U_{1}\right)<(1-\alpha) F_{X}^{-1}\left[1-\left(1-U_{Y}\right)^{\frac{1}{N-1}}\right]\right] \\
& =\mathbb{P}\left\{F_{X_{1}} F_{X_{1}}^{-1}\left(U_{1}\right)<F_{X_{1}}\left[(1-\alpha) F_{X}^{-1}\left(1-\left(1-U_{Y}\right)^{\frac{1}{N-1}}\right)\right]\right\} \\
& =\mathbb{P}\left\{U_{1}<F_{X_{1}}\left[(1-\alpha) F_{X}^{-1}\left(1-\left(1-U_{Y}\right)^{\frac{1}{N-1}}\right)\right]\right\},
\end{aligned}
$$

where the monotonic transformation $F_{X_{1}}(\cdot)$ has been used. Since both the auxiliary variables $U_{1}$ and $U_{Y}$ are uniformly distributed in $(0,1)$, and independent as well, their joint probability density function is uniform on $(0,1)^{2}$. The detection probability is then

$$
\begin{aligned}
P_{\mathrm{det}} & =\mathbb{P}\left\{U_{1}<F_{X_{1}}\left[(1-\alpha) F_{X}^{-1}\left(1-\left(1-U_{Y}\right)^{\frac{1}{N-1}}\right)\right]\right\} \\
& =\int_{0}^{1} \int_{0}^{F_{X_{1}}\left[(1-\alpha) F_{X}^{-1}\left(1-(1-v)^{N-1}\right)\right]} f_{U_{1} U_{Y}}(u, v) d u d v \\
& =\int_{0}^{1} F_{X_{1}}\left[(1-\alpha) F_{X}^{-1}\left(1-(1-v)^{\frac{1}{N-1}}\right)\right] d v \\
& =\int_{0}^{1} F_{Z}\left[\frac{(1-\alpha)\left[\mu+\sigma F_{Z}^{-1}\left(1-(1-v)^{\frac{1}{N-1}}\right)\right]-\beta \mu}{\sigma}\right] d v \\
& =\int_{0}^{1} F_{Z}\left[(1-\alpha-\beta) \frac{\mu}{\sigma}+(1-\alpha) F_{Z}^{-1}\left(1-(1-v)^{\frac{1}{N-1}}\right)\right] d v,
\end{aligned}
$$

where the standard Gaussian distribution function $F_{Z}(\cdot)$ has been used.

\section{Appendix C. Correct identification of the anomalous bid}

In Scenario B, we can take the decision $D_{1}$ in either of two cases (see Section 4.1): the bid detected as anomalous is the truly anomalous bid (case c2) or a regular bid (case w3). We show here that case w3 need not be considered, since its probability is very low.

The minimum bid $X_{(1)}$ is declared to be anomalous if the following comparison with the second lowest bid holds true

$X_{(1)}<(1-\alpha) X_{(2)}$, 
The probability $P_{\mathrm{dec}}$ of declaring the presence of anomalous bid, when it is actually present, is

$P_{\mathrm{dec}}=\mathbb{P}\left(X_{(1)}<(1-\alpha) X_{(2)} \mid\right.$ Scenario B $)$,

which can be decomposed, by the total probability theorem, in the sum

$P_{\mathrm{dec}}=\sum_{k=1}^{n} \mathbb{P}\left(X_{(1)}<(1-\alpha) X_{(2)} \mid X_{(1)}=X_{k}\right) \mathbb{P}\left(X_{(1)}=X_{k}\right)$

Now we observe that

$$
\begin{aligned}
\mathbb{P}\left(X_{(1)}\right. & \left.=X_{k}\right)=\mathbb{P}\left(X_{1}, X_{2}, \ldots, X_{k-1}, X_{k+1}, \ldots, X_{n}>X_{k}\right) \\
& =\int_{-\infty}^{\infty} \mathbb{P}\left(X_{1}, X_{2}, \ldots, X_{k-1}, X_{k+1}, \ldots, X_{n}>X_{k} \mid X_{k}\right. \\
& =x) \mathbb{P}\left(X_{k}=x\right) \\
& =\mathbb{E}_{X_{k}}\left[\mathbb{P}\left(X_{1}, X_{2}, \ldots, X_{k-1}, X_{k+1}, \ldots, X_{n}>X_{k} \mid X_{k}=x\right)\right] \\
& =\mathbb{E}_{X_{k}}\left[\prod_{j=1 j \neq k}^{n} \mathbb{P}\left(X_{j}>x\right)\right]
\end{aligned}
$$

The computation of (C.4) is different for the two cases $k=1$ and $k \geqslant 2$.

For $k=1$, it holds

$$
\begin{aligned}
\mathbb{E}_{X_{k}}\left[\prod_{j=1}^{n} \mathbb{P}\left(X_{j}>x\right)\right]= & \frac{1}{\sigma \sqrt{2 \pi}} \\
& \times \int_{-\infty}^{\infty}\left[1-F_{Z}\left(\frac{x-\mu}{\sigma}\right)\right]^{n-1} e^{-\frac{(x-\beta \mu)^{2}}{2}} d x
\end{aligned}
$$

For $k \geqslant 2$, it holds

$$
\begin{aligned}
& \mathbb{E}_{X_{k}}\left[\prod_{j=1 j \neq k}^{n} \mathbb{P}\left(X_{j}>x\right)\right]=\frac{1}{\sigma \sqrt{2 \pi}} \int_{-\infty}^{\infty} \\
& \times \frac{\left[1-F_{Z}\left(\frac{x-\mu}{\sigma}\right)\right]^{n-2}\left[1-F_{Z}\left(\frac{x-\beta \mu}{\sigma}\right)\right]}{e^{\frac{1}{2}(x-\mu)^{2}}} d x
\end{aligned}
$$

Though we could now evaluate the term $P\left(X_{(1)}=X_{k}\right)$ numerically to show that it is quite close to 1 when $k=1$, but negligible otherwise (so that the case w3 can be practically neglected), we turn to simulation to show the same result in a number of cases.

Namely, we evaluate by simulation the probability of declaring the presence of an anomalous bid $\mathbb{P}\left(X_{(1)}<(1-\alpha) X_{(2)}\right)$ and the probability of correctly identifying the anomalous bid itself $\mathbb{P}\left(X_{1}<(1-\alpha) X_{(2)}\right)$. The difference between the two is just the probability of occurrence of case w3.

We report here the results obtained with $10^{5}$ simulation runs, $\alpha=0.2$ and $\sigma / \mu=0.1$. The curves are shown in Figs. C.15 and C.16

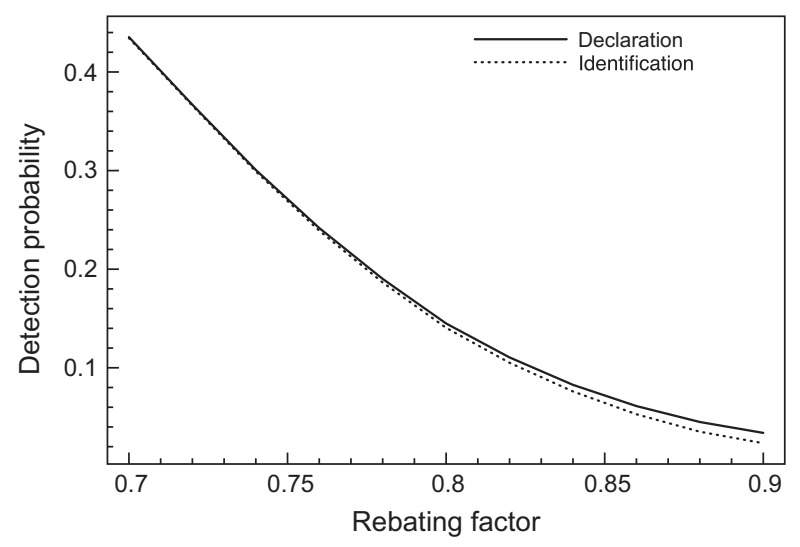

Fig. C.15. Probabilities of declaration and correct identification $(N=10)$.

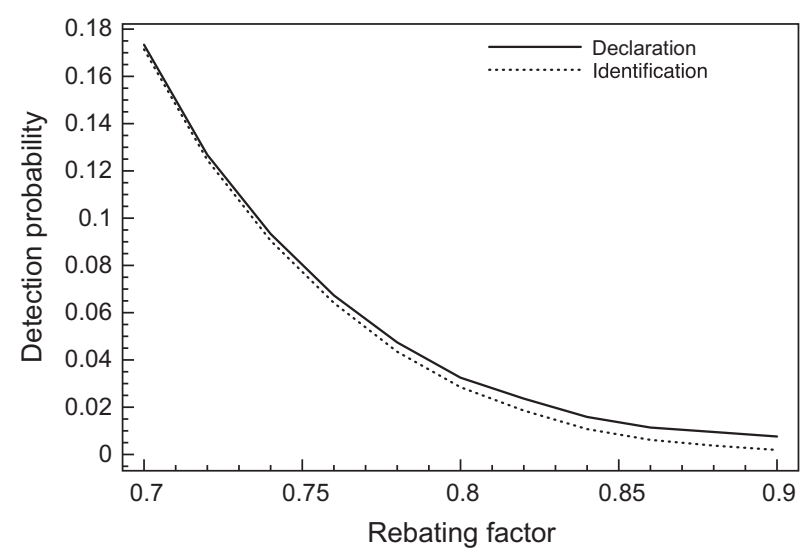

Fig. C.16. Probabilities of declaration and correct identification $(N=100)$.

for 10 and 100 bidders respectively. As can be seen, the curves are almost coincident for most values of the rebating factor, so that the declaration of the presence of an abnormally low bid is practically tantamount to its correct identification. The probability of declaration with wrong identification (case w3) becomes significant just when both the rebating factor and the number of participants are very high. But such combination is likely to lead us not to use the detection criterion anyway.

We can therefore safely conclude that the probability of declaration of the presence of an anomalous bid without its correct identification is practically negligible.

\section{References}

Akai, K., Saijo, T., and Serizawa, S. An experimental study of Japanese procurement auctions with endogenous minimum prices. The Institute of Social and Economic Research, Osaka University, Discussion Paper No. 743, 2009.

Albano, G., Dini, F., and Zampino, R. Suppliers' behavior in competitive tendering Evidence from the italian ministry of economy and finance's acquisitions of it services. In Proceedings of the Third International Public Procurement Conference, 28-30 August, Amsterdam, 2006, 667-705.

Alexandersson, G., and Hultén, S. Predatory bidding in competitive tenders: a Swedish case study. European Journal of Law Economics, 22, 2006, 73-94.

Alexandersson, G., and Hultén, S. High and low bids in tenders: strategic pricing and other bidding behaviour in public tenders of passenger railway services. Annals of Public and Cooperative Economics, 78, 2007, 161-194.

Amelinckx, I., Muylle, S., and Lievens, A. Extending electronic sourcing theory: an exploratory study of electronic reverse auction outcomes. Electronic Commerce Research and Applications, 7, 2008, 119-133.

Banker, R. D., and Mitra, S. Procurement models in the agricultural supply chain: a case study of online coffee auctions in India. Electronic Commerce Research and Applications, 6, 2007, 309-321.

Barnett, V., and Lewis, T. Outliers in Statistical Data. Wiley, New York, 1994.

Beckman, R., and Cook, R. Outlier...s. Technometrics, 25, 1983, 119-149.

Beresford-Jones, J. Abnormally low offers - too good to be true? 26 April 2010. $<$ www.lexology.com>.

Brown, K. A theoretical and statistical study of decision making under uncertainty a competitive bidding for leases of offshore petroleum tracts. Ph.D. thesis Southern Methodist University, 1966.

Casella, G., and Berger, R. Statistical Inference. Duxbury Press, 1990.

Chen, J., Xu, L., and Whinston, A. B. Managing project failure risk through contingent contracts in procurement auctions. Decision Analysis, 7, 2010, 23-39.

Conti, P. L., and Naldi, M. Detection of anomalous bids in procurement auctions. Decision Support Systems, 46, 2008, 420-428.

Davila, A., Gupta, M., and Palmer, R. Moving procurement systems to the internet: the adoption and use of e-procurement technology models. European Management Journal, 21, 2003, 11-23.

Decarolis, F. When the highest bidder loses the auction: theory and evidence from public procurement. Temi di discussione (Economic working papers) 717, Bank of Italy, Economic Research Department, 2009.

Dimitri, N., Piga, G., and Spagnolo, G. (eds.), Cambridge University Press, Cambridge, 2006, 006.

Engel, A. R. Essays on risk management in procurement auctions. Ph.D. thesis, Friedrich-Alexander-Universität Erlangen-Nürnberg, Wirtschatfs- und Sozialwissenschaftliche Fakultät, 2005.

European Court of Justice Cases C-147/06 and C-148/06. Section IV, 15 May 2008.

European Parliament Directive 2004/18/EC on the coordination of the procedures for the award of public works contracts, public supply contracts and public service contracts, 31 March 2004 
EUWG, European Union Working Group on the prevention detection and elimination of abnormally low tenders in the European construction industry abnormally low tenders, 1999.

Florida Department of Transportation Award and execution of the contract. Rev 27-97, 7-00, 1997.

Grubbs, F. Sample criteria for testing outlying observations. The Annals of Mathematical Statistics, 21, 1950, 27-58.

Gunduz, M., and Karacan, V. Damage to treasury: abnormally low tenders in public construction works. Journal of Legal Affairs and Dispute Resolution in Engineering and Construction, 1, 2009, 130-136.

Huang, H., Kauffman, R. J., Xu, H., and Zhao, L. Mechanism design for e-procurement auctions: on the efficacy of post-auction negotiation and quality effort incentives. Electronic Commerce Research and Applications, 10, 2011, 650-672.

Kobayashi, B. The law and economics of predatory pricing. George Mason University Law and Economics Research Paper Series. Paper 08-41, 2008.

McCaffer, R. Contractor's bidding behaviour and tender price prediction. Ph.D. thesis, Loughborough University of Technology, 1976.

McCaffer, R., and Pettitt, A. Distribution of bids for buildings and roads contracts Operations Research Quarterly, 27, 1976, 835-843.

Ministerio de Hacienda de España Ley de contratos de las administraciones públicas. Boletín Oficial del Estado, 148, 2000.

Ministerio de Hacienda de España Reglamento general de la ley de contratos de las administraciones públicas. Boletín Oficial del Estado, 257, 2001.

Naldi, M., and D'Acquisto, G. Performance of the Vickrey auction for digital goods under various bid distributions. Performance Evaluation, 65, 2008, 10-31.

Oren, S., and Rothkopf, M. Optimal bidding in sequential auctions. Operations Research, 23, 1975, 1080-1090.

Papazoglou, M., and Tsalgatidou, A. Business to business electronic commerce issues and solutions. Decision Support Systems, 29, 2000, 301-304.
Pin, T. H., and Scott, W. Bidding model for refurbishment work. Journal of Construction and Engineering Management, 120, 1994, 257-273.

Porter, R., Ronen, A., Shoham, Y., and Tennenholtz, M. Fault tolerant mechanism design. Artificial Intelligence, 172, 2008, 1783-1799.

Porter, R., and Shoham, Y. On cheating in sealed-bid auctions. Decision Support Systems, 39, 2005, 41-54.

Presidente della Repubblica Italiana. D. Lgs. 163/2006 Codice dei contratti pubblici relativi a lavori, servizi e forniture in attuazione delle direttive 2004/17/CE e 2004/18/CE. Suppl. ordinario n. 107 della Gazzetta Ufficiale n. 100, 2 May 2006.

Ramchurn, S. D., Mezzetti, C., Giovannucci, A., Rodríguez-Aguilar, J. A., Dash, R. K., and Jennings, N. R. Trust-based mechanisms for robust and efficient task allocation in the presence of execution uncertainty. Journal of Artificial Intelligence Research, 35, 2009, 119-159.

Sardinha, A., Benisch, M., Sadeh, N., Ravichandran, R., Podobnik, V., and Stan, M. The 2007 procurement challenge: a competition to evaluate mixed procurement strategies. Electronic Commerce Research and Applications, 8, 2009, 106-114.

Skitmore, R. Identifying non-competitive bids in construction contract auctions. OMEGA: International Journal of Management Science, 30, 2002, 443-449.

Subramaniam, C., and Shaw, M. J. A study on the value of B2B e-commerce: the case of web-based procurement. In M. J. Shaw (ed.), E-Business Management. Integrated Series in Information Systems, vol. 1, Springer, US, 2002, 439-461.

Tietjen, G. The analysis and detection of outliers. In R. D'Agostino and M. Stephens (eds.), Goodness-of-fit Techniques, Marcel Dekker, 1986.

Whittaker, J. A study of competitive bidding with particular reference to the construction industry. Ph.D. thesis, City University, London, 1970.

Zanza, M. Final presentation of the works of the CONSIP working group on auction design and competitive issues, Rome, 13 December 2004. 